\title{
A Comparison of Plain Radiography with Computer Tomography in Determining Coronal and Sagittal Alignments following Total Knee Arthroplasty
}

\author{
Solayar GN, FRCS (Tr \& Orth), Chinappa J*, MS (Orth), Harris IA**, FRACS, Chen DB***, FRACS, \\ Macdessi SJ***, FRACS \\ Department of Orthopaedics, International Medical University (IMU), Seremban, Malaysia \\ *Department of Orthopaedics, Canterbury Hospital, Sydney, Australia \\ **Ingham Institute for Applied Medical Research, South Western Sydney Clinical School, UNSW Australia, \\ Sydney, Australia \\ ***Sydney Knee Specialists, St George Private Hospital, Sydney, Australia
}

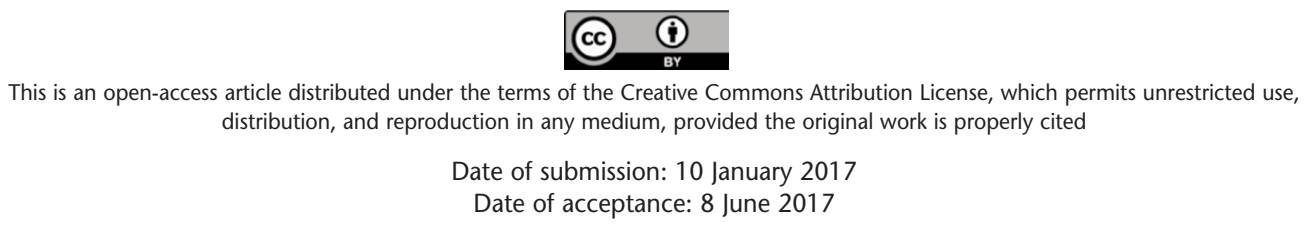

\section{ABSTRACT}

Introduction: Optimal coronal and sagittal component positioning is important in achieving a successful outcome following total knee arthroplasty (TKA). Modalities to determine post-operative alignment include plain radiography and computer tomography (CT) imaging. This study aims to determine the accuracy and reliability of plain radiographs in measuring coronal and sagittal alignment following TKA.

Materials and Methods: A prospective, consecutive study of 58 patients undergoing TKA was performed comparing alignment data from plain radiographs and CT imaging. Hipknee-angle (HKA), sagittal femoral angle (SFA) and sagittal tibial angle (STA) measurements were taken by two observers from plain radiographs and compared with CT alignment. Intra- and inter-observer correlation was calculated for each measurement.

Results: Intra-observer correlation was excellent for HKA $(r>0.89)$ with a mean difference of $<1.9^{\circ}$. The least intraobserver correlation was for SFA (mean $\mathrm{r}=0.58$ ) with a mean difference of $8^{\circ}$. Inter-observer correlation was better for HKA $(r>0.95)$ and STA $(r>0.8)$ compared to SFA $(r=0.5)$. When comparing modalities (radiographs vs CT), HKA estimations for both observers showed the least maximum and mean differences while SFA observations were the least accurate.

Conclusion: Radiographic estimation of HKA showed excellent intra- and inter-observer correlation and corresponds well with CT imaging. However, radiographic estimation of sagittal plane alignment was less reliably measured and correlated less with $\mathrm{CT}$ imaging. Plain radiography was found to be inferior to CT for estimation of biplanar prosthetic alignment following TKA.

\section{Key Words:}

radiographs, computer tomography, coronal alignment, sagittal alignment, total knee arthroplasty

\section{INTRODUCTION}

Establishing optimal mechanical alignment in total knee arthroplasty (TKA) surgery is a central component to achieving a successful outcome. It has been recommended that femoral and tibial components be positioned with less than $3^{\circ}$ of error in the coronal plane to improve functional outcomes and avoid alignment associated complications ${ }^{1}$. Optimal sagittal positioning, according to some authors, include the femoral component positioned between 0 to $3^{\circ}$ and the tibial component between 0 to $7^{\circ 2,3}$. Post-operative alignment data is often obtained using long-leg radiographs. Post-operative radiographic measurements have been reported as reliable by some authors ${ }^{4-6}$ while other studies report significant variations ${ }^{7-10}$. Other methods of determining alignment such as $\mathrm{CT}^{11}, \mathrm{MRI}^{12}$, intra-operative supine fluoroscopy ${ }^{8}$ and intra-operative navigation ${ }^{13-14}$ have been reported previously with varying results. There is evidence to suggest equivocal sensitivities between plain radiography and $\mathrm{CT}$ imaging in estimating the post-operative mechanical axis in the coronal plane ${ }^{4}$.

Most research has been dedicated to coronal alignment following TKA; less emphasis has been placed on the 
accuracy of prosthetic alignment in the sagittal plane. Sagittal alignment may influence the outcome of TKA in different ways: failure of accurate sagittal plane component positioning may result in polyethylene post wear, femoral cortical notching, limitation of motion and possible fractures ${ }^{15-16}$. Determining sagittal component alignment using plain radiographs is challenging due to the effects of femoral bowing and difficulty in establishing a true sagittal axis using standard lateral radiographs of the knee that also include the hip and ankle joint. To the best of our knowledge, there are no studies correlating the accuracy of plain radiography versus $\mathrm{CT}$ in the sagittal plane.

The benefits of plain radiography include its availability, affordability, and the ability to detect the influence of weight-bearing on lower limb alignment. Limitations of radiographs are potential inaccuracy stemming from alterations in patient positioning and certain anatomic configurations (rotation and flexion contractures in particular) as described by some authors ${ }^{17-18}$. A full length lateral radiograph of the femur from hip to knee centres is difficult to obtain and as such a true sagittal mechanical axis determination is usually not possible. This measurement is often estimated from anatomic axis projections extending proximally from the knee joint for varying distances depending on the length of the $\mathrm{x}$-ray plate. Plain radiographs are also unable to measure rotational alignment of the femoral and tibial implants.

Computer tomography may improve accuracy by negating the effects of patient position and improve detection of anatomic landmarks in all three planes (axial, coronal and sagittal). Full length mechanical axis determination is possible for both the femur and the tibia. Limitations of CT include the inability of incorporating weight-bearing and the higher cost. In addition, the effect of radiation exposure is a concern when using CT imaging. Radiation dose is generally quoted as the weighted dose $(\mathrm{mSv})$ received by the body's radiosensitive organs. The Perth protocol lower limb CT scans (which was used in this study) gives a dose of $2.7 \mathrm{mSv}^{19}$. In comparison, a standard long-leg standing radiograph gives a dose of about $0.7 \mathrm{mSv}^{11}$. The average Australian background radiation was around $2 \mathrm{mSv}$ per year $^{20}$.

The objective of this study was to investigate the accuracy and reliability of plain radiographs in measuring coronal and sagittal alignment following TKA, using CT imaging as the gold standard. If measurements from plain radiographs could be shown to be accurate, CT tomography could then be limited to assessing rotational positioning of knee components. This could potentially result in a decrease in radiation exposure to the patient as well as reducing costs incurred for this imaging.

\section{MATERIALS AND METHODS}

We performed a prospective study of 62 consecutive patients who underwent TKA at our institution between December 2014 and April 2015. Four patients were excluded due to incomplete or insufficient radiographs. The remaining 58 patients were included in this study. Demographics of the study population are summarised in Table I. Pre-operative hip-knee angle (HKA) was measured in all patients. Postoperative CT imaging and plain radiographs were evaluated and analysed. This study was approved by the institutional ethics committee.

All surgeries were performed by two fellowship-trained orthopaedic surgeons. All patients received a cemented, posterior-stabilised, total condylar knee arthroplasty (Legion $^{\mathrm{TM}}$, Smith \& Nephew). A medial para-patellar approach was utilised and all operations were performed using a measured resection technique. We aimed for restoration of a neutral hip-knee angle in the coronal plane, 3 degrees of femoral component flexion in the sagittal plane and 3 degrees posterior tibial slope.

All patients in this series underwent post-operative plain radiographs using digital imaging. This included a 4-foot long standing film of the lower limb as well as an 18-inch, non-weight bearing lateral radiographs of the operated knee on the second day post-operatively (Fig. 1). The 4-foot films were used to assess coronal plane alignment and the 18-inch lateral film was chosen to optimise the chance of determining the true sagittal femoral and tibial mechanical alignment based on anatomic axis projections. For lateral radiographs, the patient's foot was placed in a positioner which allowed for knee flexion between $30^{\circ}$ to $45^{\circ}$. Limb rotation was deemed satisfactory if there was complete overlap of the medial and lateral femoral condyles on the lateral radiograph. Component alignments were evaluated, as described by Hsu et $a l^{21-22}$ to determine, (1) the hip-knee angle (HKA) determined by the mechanical axes of both the femur and the tibia, (2) the sagittal femoral angle (SFA) determined between the sagittal anatomic distal femoral axis and the perpendicular axis of the femoral component and (3) the sagittal tibial angle (STA) between the sagittal anatomic proximal tibial axis and the perpendicular axis of the tibial component. Target surgical alignment values were a HKA of $0^{\circ}$ in the coronal plane, a SFA of $3^{\circ}$ for the femoral component and a STA of $3^{\circ}$ of posterior slope for the tibial component.

In addition, all patients underwent a low dose CT scan using the Perth CT Protocol ${ }^{19}$. This technique allows non-weight bearing measurements of the femoral and tibial component alignments in the coronal, sagittal and axial plains with the added benefit of assessing rotational positioning. The anatomical landmarks determined included the centre of the femoral head, the centre of the talus, mid-point of the 
Table I: Demographics and pre-operative details

\begin{tabular}{|lc|}
\hline Parameter & Study Group \\
\hline Age* (years) & $66.4 \pm 7.8(47-88)$ \\
Involved knee (R/L) & $32 / 27$ \\
Sex (F/M) & $33 / 26$ \\
Alignment method (Navigated/IM) & $14 / 44$ \\
Pre-op Hip-Knee Angle * & $-2.65^{\circ} \pm 6.53^{\circ}\left(-13^{\circ}\right.$ to $\left.13^{\circ}\right) * *$ \\
\hline
\end{tabular}

*Values are shown as the mean and standard deviation with the range in parentheses.

**Positive values denote a valgus angle; Negative values denote varus angulature.

Table II: Intra-observer correlation by radiographic measurement

\begin{tabular}{|c|c|c|c|c|c|c|c|c|c|}
\hline & \multicolumn{3}{|c|}{ Hip-Knee Angle } & \multicolumn{3}{|c|}{ Sagittal Femoral Angle } & \multicolumn{3}{|c|}{ Sagittal Tibial Angle } \\
\hline & $\begin{array}{l}\text { Maximum } \\
\text { difference }\end{array}$ & $\begin{array}{c}\text { Mean } \\
\text { difference }\end{array}$ & $\mathrm{SD}^{*}$ & $\begin{array}{l}\text { Maximum } \\
\text { difference }\end{array}$ & $\begin{array}{c}\text { Mean } \\
\text { difference }\end{array}$ & SD* & $\begin{array}{l}\text { Maximum } \\
\text { difference }\end{array}$ & $\begin{array}{c}\text { Mean } \\
\text { difference }\end{array}$ & SD* \\
\hline Observer 1 & $3.2^{\circ}$ & $0.66^{\circ}$ & 0.56 & $6^{\circ}$ & $1.47^{\circ}$ & 1.16 & $3.5^{\circ}$ & $1.21^{\circ}$ & 0.94 \\
\hline Observer 2 & $2.6^{\circ}$ & $0.71^{\circ}$ & 0.6 & $12.1^{\circ}$ & $2.61^{\circ}$ & 2.58 & $6.4^{\circ}$ & $1.69^{\circ}$ & 1.34 \\
\hline
\end{tabular}

*Standard Deviation

Table III: Inter-observer correlation by radiographic measurements

\begin{tabular}{|c|c|c|c|c|c|c|c|c|}
\hline \multicolumn{3}{|c|}{ Hip-Knee Angle } & \multicolumn{3}{|c|}{ Sagittal Femoral Angle } & \multicolumn{3}{|c|}{ Sagittal Tibial Angle } \\
\hline $\begin{array}{l}\text { Maximum } \\
\text { difference }\end{array}$ & $\begin{array}{c}\text { Mean } \\
\text { difference }\end{array}$ & SD* & $\begin{array}{l}\text { Maximum } \\
\text { difference }\end{array}$ & $\begin{array}{c}\text { Mean } \\
\text { difference }\end{array}$ & SD* $^{*}$ & $\begin{array}{l}\text { Maximum } \\
\text { difference }\end{array}$ & $\begin{array}{c}\text { Mean } \\
\text { difference }\end{array}$ & SD* $^{*}$ \\
\hline $1.65^{\circ}$ & $0.54^{\circ}$ & 0.4 & $10.7^{\circ}$ & $2.37^{\circ}$ & 2.02 & $3.2^{\circ}$ & $0.93^{\circ}$ & 0.76 \\
\hline
\end{tabular}

*Standard Deviation

Table IV: Inter-modality correlation between radiographs and CT for each observer

\begin{tabular}{|c|c|c|c|c|c|c|c|c|c|}
\hline & \multicolumn{3}{|c|}{ Hip-Knee Angle } & \multicolumn{3}{|c|}{ Sagittal Femoral Angle } & \multicolumn{3}{|c|}{ Sagittal Tibial Angle } \\
\hline & $\begin{array}{l}\text { Maximum } \\
\text { difference }\end{array}$ & $\begin{array}{c}\text { Mean } \\
\text { difference }\end{array}$ & SD* $^{*}$ & $\begin{array}{l}\text { Maximum } \\
\text { difference }\end{array}$ & $\begin{array}{c}\text { Mean } \\
\text { difference }\end{array}$ & $\mathrm{SD}^{*}$ & $\begin{array}{l}\text { Maximum } \\
\text { difference }\end{array}$ & $\begin{array}{c}\text { Mean } \\
\text { difference }\end{array}$ & SD* \\
\hline Observer 1 & $6^{\circ}$ & $1.37^{\circ}$ & 1.11 & $8.5^{\circ}$ & $1.79^{\circ}$ & 1.49 & $4.4^{\circ}$ & $1.45^{\circ}$ & 1.02 \\
\hline Observer 2 & $5.4^{\circ}$ & $1.09^{\circ}$ & 0.99 & $12.85^{\circ}$ & $2.63^{\circ}$ & 2.62 & $3.55^{\circ}$ & $1.3^{\circ}$ & 0.97 \\
\hline
\end{tabular}

*Standard Deviation

intercondylar notch and centre of the polyethylene insert or tibial plateau.

The CT tube current was set at $120 \mathrm{kV}$ with an effective mAs of 50-70. Helical scans covered from just above the acetabulum to just below the ankle joint with image reconstructions of $2 \mathrm{~mm}$ thickness at $1.6 \mathrm{~mm}$ intervals. The limb was positioned with the knee in full extension and rotated in to the AP position. All measurements were performed on a Siemens workstation. Selected images were then chosen from the $3 \mathrm{D}$ tab and then linked to mark the centre of each region on all images. This technique allowed accurate reformatting of the limb in the true frontal, sagittal and axial planes so that rotational positional errors were minimised. Our institutions chief radiographer performed all CT scans and undertook measurements of HKA, SFA and STA (Fig. 2).
All standard radiographic measurements were independently performed by two observers, one an orthopaedic surgeon (GS) and the other an orthopaedic resident (JC). CT radiographic measurements were performed by the chief radiographer (NT) at our institution with CT Perth Protocol measurement experience over 5 years. The syngo CT2012a ${ }^{\circledR}$ software (Siemens Healthcare $\mathrm{GmbH}$ ) was utilised for all radiographic and $\mathrm{CT}$ measurements.

Statistical analyses were performed using Microsoft Excel 2013 (Microsoft Corp., Redmond, WA, USA) software and MedCalc ${ }^{\circledR}$ version 15.4 (C) 1993-2015 MedCalc Software bvba, Ostend, Belgium). The Pearson Product Moment Correlation test was used to determine intra- and interobserver alignment measurements of post-operative radiographs (HKA, SFA and STA). Bland-Altman plots were utilised to assess agreement and are represented graphically. 
The 95\% limits of agreement between observers are defined as the mean difference plus and minus 1.96 times the standard deviation of the differences ${ }^{23}$. Coefficient of repeatability was defined as two standard deviations of the difference between measurement and was a good indication of repeatability.

\section{RESULTS}

When measurements obtained by each of the observers were compared, an excellent correlation was found for each observer looking at HKA using long-leg radiographs ( $\mathrm{r}=0.92$ and $r=0.89$ ) with a mean difference of $-0.03^{\circ}$ (range -1.74 to $1.66^{\circ}$ ) and $-0.16^{\circ}$ (range -1.96 to $1.65^{\circ}$ ) for each observer. It was noted that the correlation reduced for both observers when measuring for SFA $(r=0.67$ and $r=0.51)$ and STA $(\mathrm{r}=0.75$ and $\mathrm{r}=0.47)$. The mean difference for measuring SFA was $-0.07^{\circ}$ (range -3.79 to $3.66^{\circ}$ ) and $-2.04^{\circ}$ (range -8.03 to $3.94^{\circ}$ ) for each observer while the mean difference for STA was $0.21^{\circ}$ (range -2.76 to $3.2^{\circ}$ ) and $-0.72^{\circ}$ (range -4.72 to $3.29^{\circ}$ ) (Table II).

When measuring for HKA, an excellent inter-observer correlation was found with $\mathrm{r}=0.96$ (range 0.93-0.97, $\mathrm{p}<0.0001$ ) with a mean difference of $0.3^{\circ}$. The correlation figures were lower for STA $(r=0.81$, range 0.7 to 0.88 , $\mathrm{p}<0.0001)$ and the mean difference was $-0.1^{\circ}$. Measuring SFA provided the worst inter-observer correlation SFA with $\mathrm{r}=0.5$ (range $0.28-0.67, \mathrm{p}=0.0001$ ) and a mean difference of $1.95^{\circ}$ (Table III). Graphical representation to indicate agreement for inter-observer measurements are provided in Figure 3.

For Observer 1, the correlation between radiographic HKA, SFA and STA measurements compared to CT results were $\mathrm{r}=0.75(\mathrm{p}<0.0001), \mathrm{r}=0.18(\mathrm{p}=0.19)$ and $\mathrm{r}=0.66(\mathrm{p}<0.0001)$ respectively. For Observer 2, the HKA, SFA and STA correlations were $r=0.79(p<0.0001), r=-0.10(p=0.44)$ and $\mathrm{r}=0.66(\mathrm{p}<0.0001)$ respectively. (Table IV)

Graphical representations to indicate agreement between modalities for both observers are provided in Figures 4 and 5 .

\section{DISCUSSION}

In this study, we found good to excellent intra- and interobserver correlation when measuring for HKA, and to a lesser extent, STA. More importantly, these alignment values also showed reliable inter-modality correlation between observers with better comparisons for HKA over STA when comparing radiographic results to CT. Our results with regards to HKA is in keeping with prior literature comparing long-leg radiographs and $\mathrm{CT}$. Babazadeh et $a l^{4}$ showed excellent inter-observer correlation of $r>0.96$ and a mean discrepancy between observers of $0.56^{\circ}$. SFA measurements however showed inferior correlation and reproducibility compared to HKA and STA. This questions the validity and reliability of radiographic SFA evaluations when determining sagittal alignment post total knee replacement overall.

Analysing maximum and mean intra- and inter-observer differences gives an idea of the degree of error in these measurements. This error is least pronounced when measuring for HKA while the largest discrepancies were noted when evaluating SFA radiographically. When assessing for agreement between radiographic to $\mathrm{CT}$ alignment data, the inaccuracy of SFA measurements were emphasised. For both observers, the greatest maximal error, greatest mean error and largest standard deviation were all found when comparing SFA data between modalities. Agreement between radiographic HKA and STA measurements versus CT were clinically better.

There is contrasting evidence in the current literature regarding the accuracy of radiographic measurements of limb alignment following total knee replacement surgery. Lower limb flexion and rotation may give rise to inaccurate coronal alignment readings as shown previously by Brouwer $^{9}$ and Radtke ${ }^{17}$. Other authors reported poor correlation with the use of non-standardised alignment measures (using anatomical rather than the mechanical axis) and with the use of short-leg films ${ }^{7,24}$. We used standardised radiographic protocols controlling for rotation and flexion at our unit of which we believe, minimises these potential inaccuracies. The use of long-leg radiographs, as in our study, has been shown by Specogna ${ }^{6}$ to be accurate to within $0.9^{\circ}$ for coronal measurements.

The main finding in our study was the poor radiological determination of sagittal femoral component alignment using plain radiographs. Though our study employed the use of 18-inch lateral radiographs, we felt that the effect of anterior femoral bowing was significant in giving rise to poor intra- and inter- observer correlation and agreement. Furthermore, there remained a high variability in the geometry of femoral bowing within any given population resulting in difficulties in establishing clear radiological protocols when analysing sagittal component alignment ${ }^{25}$. In a study by Chung $^{26}$ et al, they identified femoral sagittal discrepancies between the distal anatomical axes versus true mechanical axes of between $5.6^{\circ}$ to $8.5^{\circ}$. They also showed that over $20 \%$ of their subjects differed more than 2 degrees from the mean value ${ }^{26}$.

Sagittal tibial component measurements in our study showed less discrepancies between radiographic modalities and better intra-/inter- observer correlation and agreement compared to SFA. This may be secondary to better anatomic relationship between proximal tibial anatomic axis projection and the tibial sagittal mechanical axis. Two separate anatomical studies by Han et al and Tsukeoka et al 
Total Knee Arthroplasty

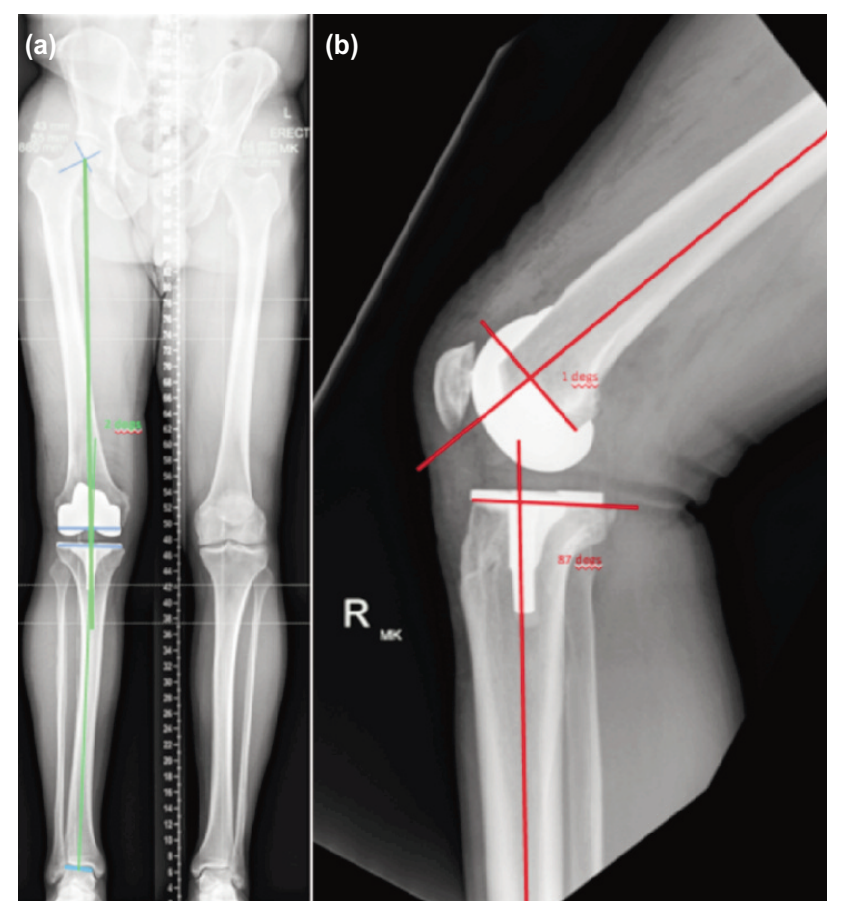

Fig. 1: (a) Post-operative measurements of Hip-Knee Angle on 4-foot standing films and (b) Sagittal Femoral Angle and Sagittal Tibial Angle on 18-inch long lateral radiographs.

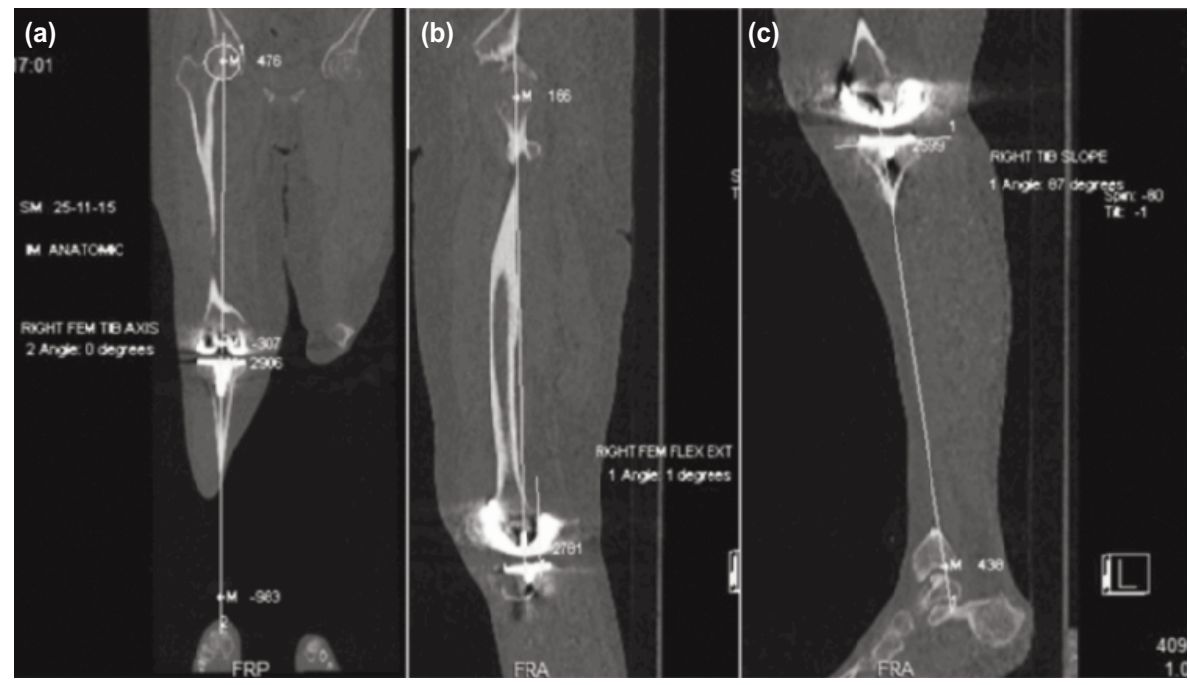

Fig. 2: Post-operative measurements of (a) Hip-Knee Angle, (b) Sagittal Femoral Angle and (c) Sagittal Tibial Angle on CT radiographs using the CT Perth Protocol.
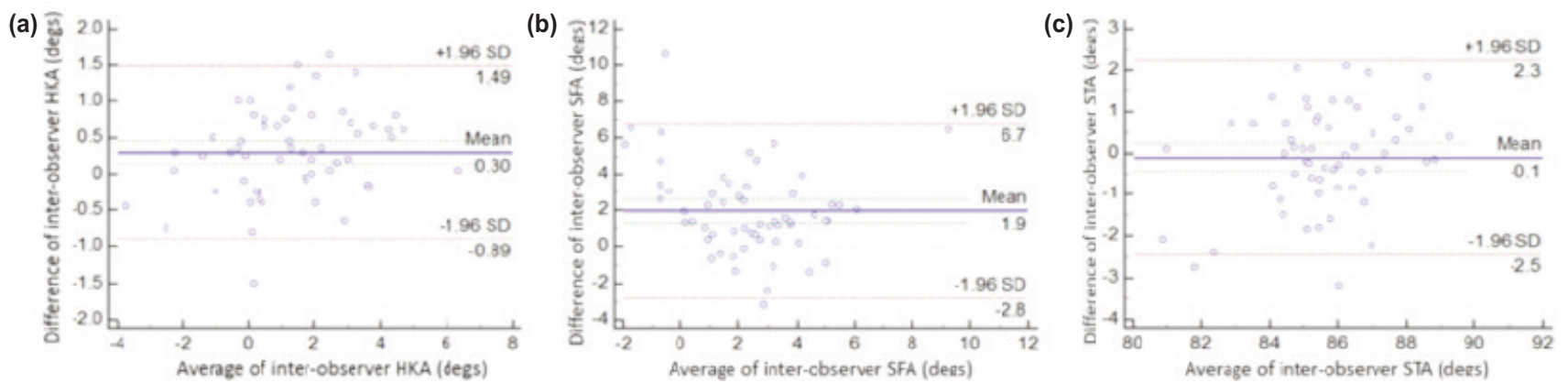

Fig. 3: Relationship between the difference and mean of inter-observer radiographic measurements for (a) Hip-Knee Angle, (b) Sagittal Femoral Angle and (c) Sagittal Tibial Angle. 
(a)

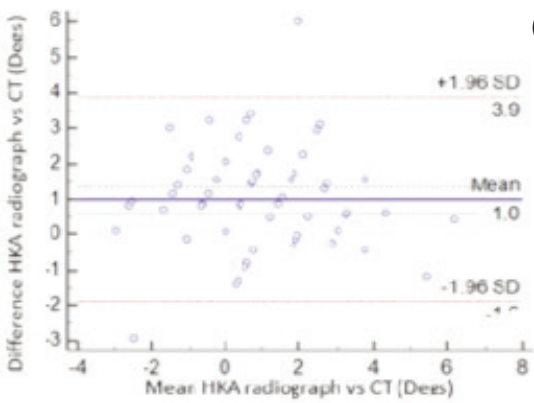

(b)

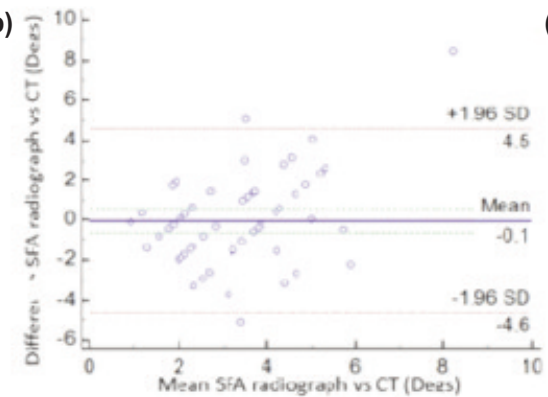

(c)

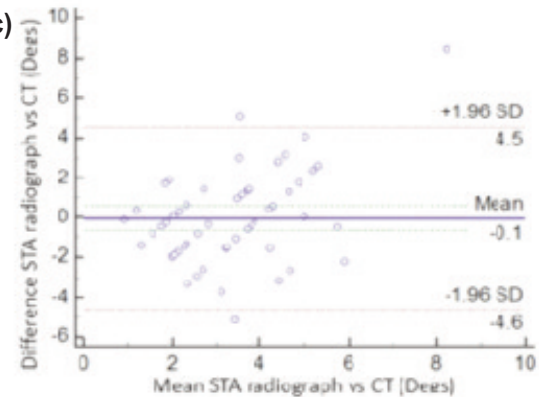

Fig. 4: Relationship between difference and mean values of alignment as measured via radiographs and CT by the first observer for (a) Hip-Knee Angle, (b) Sagittal Femoral Angle and (c) Sagittal Tibial Angle.

(a)

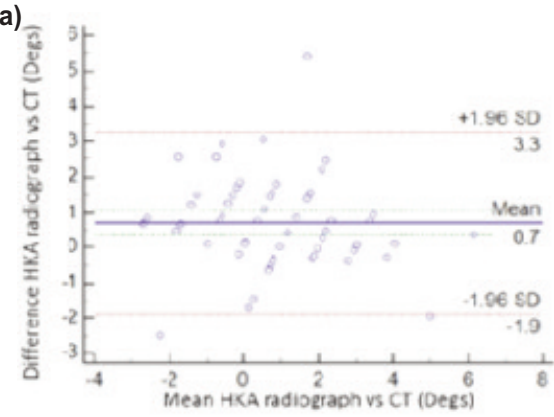

(b)

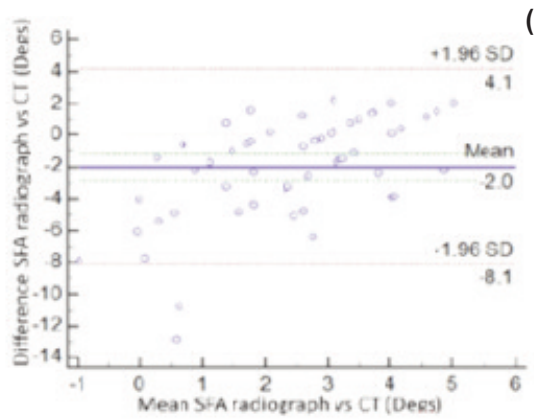

(c)

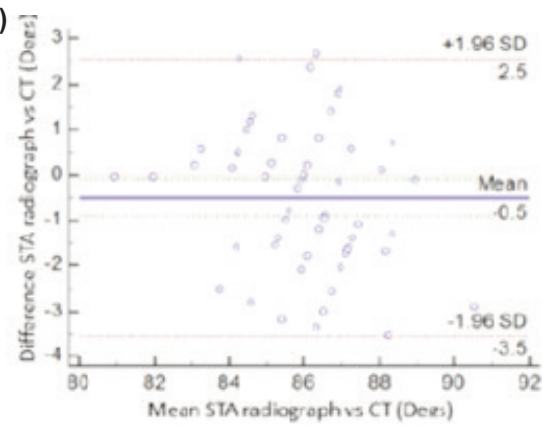

Fig. 5: Relationship between difference and mean values of alignment as measured via radiographs and CT by the second observer for (a) Hip-Knee Angle, (b) Sagittal Femoral Angle and (c) Sagittal Tibial Angle.

have shown the relationship between the anatomic and mechanical axes to be $2.2^{\circ} \pm 0.9^{27-28}$. This consistency potentially explains the difference in reliability between femoral and tibial component alignment measurements in the sagittal plane using plain radiographs.

Discrepancies in correlation and agreeability between HKA and SFA measurements in our study possibly stemmed from the availability of distinct reference points. In determining the coronal mechanical axis using long-leg radiographs, the centre of the femoral head to the centre of the ankle was often clearly demarcated for measuring alignment. In contrast, the lack of image quality often accompanying cross-table lateral films ${ }^{29}$ makes estimating the femoral head centre difficult and may therefore, reduce accuracy and reproducibility when determining the sagittal femoral mechanical axis. CT imaging would therefore be important in improving the sensitivity and specificity of sagittal femoral component position measurements.

There were several limitations to our study. We took CT data as the standard to compare our inter-modality results while in actual fact, this did not prove that CT was absolutely accurate $^{30}$. Our methods in standardising lateral radiographs could have been improved by employing longer films (including cross-table laterals) and more complex limb positioning devices that allowed for a true assessment of the sagittal mechanical axes. There remains potential discrepancies between data obtained by weight-bearing anterior-posterior four foot films compared to non-weightbearing CT results.

The purpose of this study was to determine whether conventional lower limb CT imaging following TKA to assess multiplanar knee alignment could be replaced with a coronal and sagittal plain radiography protocol and limited CT imaging of the knee for rotational component positioning. We found that long-leg radiographs remain an excellent tool in determining overall coronal component alignment and to a lesser degree, tibial component sagittal alignment. Radiographs remain a practical measurement method in terms of ease of access coupled with improved cost-effectiveness. However, we reject the study hypothesis that plain radiography is equivalent to $\mathrm{CT}$ imaging in accurately determining sagittal plane alignment of the femoral component following TKA. As such, we believe that plain radiography is inferior to $\mathrm{CT}$ in assessing postoperative biplanar knee alignment, and CT imaging remains the optimal investigation for precise multiplanar knee alignment.

Further studies looking at the effect of limb rotation on biplanar component alignment measurements using radiographs and $\mathrm{CT}$ imaging may improve our understanding regarding the merits of these radiographic modalities following total knee arthroplasty. 


\section{REFERENCES}

1. Jeffery RS, Morris RW, Denham RA. Coronal alignment after total knee replacement. J Bone Joint Surg Br. 1991; 73(5): 709-14.

2. Kim YH, Park JW, Kim JS, Park D. The relationship between the survival of total knee arthroplasty and postoperative coronal, sagittal and rotational alignment of knee prosthesis. Int Orthop. 2014; 38(2): 379-85.

3. Lustig S, Scholes CJ, Stegeman TJ, Oussedik S, Coolican MRJ, Parker DA. Sagittal placement of the femoral component in total knee arthroplasty predicts knee flexion contracture at one-year follow-up. Int Orthop. 2012; 36(9): 1835-9.

4. Babazadeh S, Dowsey MM, Bingham RJ, Ek ET, Stoney JD, Choong PFM. The long leg radiograph is a reliable method of assessing alignment when compared to computer-assisted navigation and computer tomography. Knee. 2013; 20(4): 242-9.

5. Rauh MA, Boyle J, Mihalko WM, Phillips MJ, Bayers-Thering M, Krackow KA. Reliability of measuring long-standing lower extremity radiographs. Orthopedics. 2007; 30(4): 299-303.

6. Specogna AV, Birmingham TB, DaSilva JJ, Milner JS, Kerr J, Hunt MA, et al. Reliability of lower limb frontal plane alignment measurements using plain radiographs and digitized images. J Knee Surg. 2004; 17(4): 203-10.

7. Schmidt GL, Altman GT, Dougherty JT, DeMeo PJ. Reproducibility and reliability of the anatomic axis of the lower extremity. J Knee Surg. 2004; 17(3): 141-3.

8. Sabharwal S, Zhao C. Assessment of lower limb alignment: supine fluoroscopy compared with a standing full-length radiograph. J Bone Joint Surg Am. 2008; 90(1): 43-51.

9. Brouwer RW, Jakma TSC, Brouwer KH, Verhaar JAN. Pitfalls in determining knee alignment: a radiographic cadaver study. $J$ Knee Surg. 2007; 20(3): 210-5.

10. Petersen TL, Engh GA. Radiographic assessment of knee alignment after total knee arthroplasty. J Arthroplasty. 1988; 3(1): 6772.

11. Henckel J, Richards R, Lozhkin K, Harris S, Rodriguez y Baena FM, Barrett ARW, et al. Very low-dose computed tomography for planning and outcome measurement in knee replacement. The imperial knee protocol. J Bone Joint Surg Br. 2006; 88(11): 1513-8.

12. Hinterwimmer S, Graichen H, Vogl TJ, Abolmaali N. An MRI-based technique for assessment of lower extremity deformitiesreproducibility, accuracy, and clinical application. Eur Radiol. 2008; 18(7): 1497-505.

13. Pitto RP, Graydon AJ, Bradley L, Malak SF, Walker CG, Anderson IA. Accuracy of a computer-assisted navigation system for total knee replacement. J Bone Joint Surg Br. 2006; 88(5): 601-5.

14. Yaffe MA, Koo SS, Stulberg SD. Radiographic and Navigation Measurements of TKA Limb Alignment Do Not Correlate. Clin Orthop Relat Res. 2008; 466(11): 2736-44.

15. Li G, Papannagari R, Most E, Park SE, Johnson T, Tanamal L, et al. Anterior tibial post impingement in a posterior stabilized total knee arthroplasty. J Orthop Res. 2005; 23(3): 536-41.

16. Stoller AP, Johnson TS, Popoola OO, Humphrey SM, Blanchard CR. Highly crosslinked polyethylene in posterior-stabilized total knee arthroplasty: in vitro performance evaluation of wear, delamination, and tibial post durability. J Arthroplasty. 2011; 26(3): 483-91.

17. Radtke K, Becher C, Noll Y, Ostermeier S. Effect of limb rotation on radiographic alignment in total knee arthroplasties. Arch Orthop Trauma Surg. 2010; 130(4): 451-7.

18. Lonner JH, Laird MT, Stuchin SA. Effect of rotation and knee flexion on radiographic alignment in total knee arthroplasties. Clin Orthop Relat Res. 1996; (331): 102-6.

19. Chauhan SK, Clark GW, Lloyd S, Scott RG, Breidahl W, Sikorski JM. Computer-assisted total knee replacement. A controlled cadaver study using a multi-parameter quantitative CT assessment of alignment (the Perth CT Protocol). J Bone Joint Surg Br. 2004; 86(6): 818-23.

20. Australian Radiation Protection and Nuclear Safety Agency (ARPANSA). Flying and Health - Cosmic Radiation Exposure for Casual Flyers and Aircrew. http://wwww.arpansa.gov.au/factsheets/FlyingandHealth.pdf. Accessed 22 Nov 2016 
21. Hsu RW, Himeno S, Coventry MB, Chao EY. Normal axial alignment of the lower extremity and load-bearing distribution at the knee. Clin Orthop Relat Res. 1990; (255): 215-27.

22. Ewald FC. The Knee Society total knee arthroplasty roentgenographic evaluation and scoring system. Clin Orthop Relat Res. 1989; (248): 9-12.

23. Bland JM, Altman DG. Statistical methods for assessing agreement between two methods of clinical measurement. Lancet. 1986; 1(8476): 307-10.

24. Prakash U, Wigderowitz CA, McGurty DW, Rowley DI. Computerised measurement of tibiofemoral alignment. J Bone Joint Surg Br. 2001; 83(6): 819-24.

25. Buford WL, Turnbow BJ, Gugala Z, Lindsey RW. Three-dimensional computed tomography-based modeling of sagittal cadaveric femoral bowing and implications for intramedullary nailing. J Orthop Trauma. 2014; 28(1): 10-6.

26. Chung BJ, Kang YG, Chang CB, Kim SJ, Kim TK. Differences between sagittal femoral mechanical and distal reference axes should be considered in navigated TKA. Clin Orthop Relat Res. 2009; 467(9): 2403-13.

27. Tsukeoka T, Lee TH, Tsuneizumi Y, Suzuki M. The tibial crest as a practical useful landmark in total knee arthroplasty. Knee. 2014; 21(1): 283-9.

28. Han HS, Chang CB, Seong SC, Lee S, Lee MC. Evaluation of anatomic references for tibial sagittal alignment in total knee arthroplasty. Knee Surg Sports Traumatol Arthrosc. 2008; 16(4): 373-7.

29. Bragdon CR, Martell JM, Estok DM, Greene ME, Malchau H, Harris WH. A new approach for the Martell 3-D method of measuring polyethylene wear without requiring the cross-table lateral films. J Orthop Res. 2005; 23(4): 720-5.

30. Jazrawi LM, Birdzell L, Kummer FJ, Di Cesare PE. The accuracy of computed tomography for determining femoral and tibial total knee arthroplasty component rotation. J Arthroplasty. 2000; 15(6): 761-6. 